\title{
Risk factors for development of flucloxacillin associated jaundice
}

\author{
Christopher K Fairley, John J McNeil, Paul Desmond, Richard Smallwood, Hilary Young, \\ Andrew Forbes, Patrick Purcell, Ian Boyd
}

\section{Abstract}

Objectives-To identify risk factors predisposing to the development of flucloxacillin associated jaundice.

Design-Case-control study. Medical records of cases and controls were reviewed and information recorded on standard data collection forms.

Setting-Alfred Hospital recruiting subjects from Melbourne, Sydney, and Brisbane.

Subjects-Cases were defined as patients who had developed jaundice within eight weeks of stopping flucloxacillin, biochemical test results suggesting cholestasis, normal calibre bile ducts, and not been taking recognised hepatotoxic drugs. 51 of the 53 patients referred were included in the study. Four controls for each case were randomly selected from the patient register of the prescribing doctor. These were defined as patients who had been prescribed flucloxacillin without developing jaundice.

Main outcome measures-Demographic characteristics, medical history, indication for flucloxacillin, dose, route and duration of treatment, other drugs, smoking, and previous drug allergies or use of flucloxacillin.

Results-Increasing age and a prolonged duration of flucloxacillin treatment were found to be risk factors for the development of jaundice. Patients aged over 55 years had an odds ratio of $18.61(95 \%$ confidence interval $5 \cdot 16-67 \cdot 17$ ) compared with patients under 30 . The odds ratio for patients prescribed flucloxacillin for over 14 days was $7 \cdot 13$ (2.90 to 17.58$)$ compared with patients treated for 14 days or less. Dose and route of administration were not related to the risk of jaundice.

Conclusions-Older patients and those receiving flucloxacillin for longer than two weeks are at a substantially greater risk of jaundice. Careful consideration of the risk-benefit ratio is required when flucloxacillin is used in these settings.

\section{Introduction}

Flucloxacillin is a semisynthetic penicillin that is useful for treating infections with methicillin sensitive staphylococci that produce penicillinase. Cholestatic jaundice associated with the use of this drug is rare but often severe. Jaundice often persists for more than three months and abnormal liver function may persist for 12 months or more. ${ }^{2}$

Damage to the liver associated with flucloxacillin was first reported in $1982 .{ }^{3}$ Since then further cases have been reported in Australia (131 cases), Sweden (48 cases), and the United Kingdom (21 cases). ${ }^{45}$ In Australia the incidence of liver damage has paralleled the increased use of flucloxacillin. We conducted a retrospective case-control study to determine whether any defined group of patients is at increased risk of developing flucloxacillin associated jaundice.

\section{Methods}

A case definition was established after reviewing previous reports to the Australian Adverse Drug Reaction Advisory Committee. Cases were eligible for inclusion in the study if they met the following criteria: jaundice occurred within eight weeks of the patient stopping flucloxacillin, results of liver function tests suggested cholestasis, bile ducts were normal diameter by ultrasonography or endoscopic retrograde cholangiopancreatography, patient had no other recognised causes of jaundice of hepatotoxic drugs.

Cases were recruited to the study from two sources. The first was the adverse drug reactions section of the Commonwealth (Australian) Department of Health, Housing, and Community Services. This section receives spontaneous reports of adverse drug reactions from hospitals, doctors, and pharmacists. An algorithm is used to classify reports according to the likely mechanism (idiosyncrasy, pharmacological action, etc) and the likelihood of the specified drug having caused the adverse reaction - that is, certain, probable, possible, and unlikely. Cases classified as unlikely were not used in this study. All drugs prescribed within an appropriate time frame are considered as possible causes with the term "sole suspected drug" being confined to cases where only a single drug has been prescribed before the onset of the suspected reaction.

Staff from the adverse drug reaction section wrote to 82 Australian doctors who had reported cases, requesting permission to pass their names on to the study centre. Fifty one doctors responded and 26 were selected because they lived within a convenient distance from the study centre (that is, in metropolitan Melbourne, Sydney, or Brisbane and the state of Victoria). These doctors were then contacted and asked to obtain permission for the study to contact the doctor who prescribed flucloxacillin to the patient. Twenty six prescribing doctors were subsequently contacted and all made their records available to the investigation. Twenty four of the patients identified fulfilled the inclusion criteria for the study.

We also obtained cases from a specifically established surveillance programme operated by hepatologists in Melbourne, a city of 3 million people. Between October 1991 and March 199227 new cases of jaundice meeting the case definition were identified and included in the case series. The prescribing doctor was then identified and the above protocol followed.

When the case was prescribed flucloxacillin by a private medical practitioner, the study's research nurse attended the practitioner's office and selected controls from the patient record system. This was done by progressing forwards (alphabetically) from the case record until four patients who had been prescribed flucloxacillin without developing jaundice had been identified. When the prescribing doctor was employed by a hospital a similar procedure was conducted with hospital records. 
DATA RECORDING AND ANALYSIS

Information from the medical record was entered on to a standard data extraction form by a research nurse. Care was taken to exclude information recorded after the time the flucloxacillin was prescribed. This was to avoid bias resulting from additional information being sought about potential hepatotoxins (for example, alcohol) once jaundice had been diagnosed.

Information was recorded about demography, medical history, indication for flucloxacillin, dose, route and duration of treatment, other treatments, smoking, and previous drug allergies or use of flucloxacillin.

After being checked for completeness, the data were entered on to a relational database and analysed with the EGRET statistical software package. Conditional logistic regression of the cases and matched controls was used to generate odds ratios for factors potentially associated with the development of jaundice. Potential interactions between factors were studied by including

TABLE I-Characteristics of patients with flucloxacillin associated jaundice. Values are means unless stated otherwise

\begin{tabular}{|c|c|c|c|c|}
\hline Variable & $\begin{array}{c}\text { All cases } \\
(n=51)\end{array}$ & $\begin{array}{c}\text { Patients } \\
\text { identified by } \\
\text { ADRAC } \\
(n=24)\end{array}$ & $\begin{array}{c}\text { Patients } \\
\text { identified by } \\
\text { surveillance } \\
\text { programme } \\
(n=27)\end{array}$ & $\begin{array}{c}\text { Patients in whom } \\
\text { flucloxacillin was } \\
\text { sole suspected } \\
\text { drug } \\
(n=10)\end{array}$ \\
\hline Mean age (years) & $60 \cdot 1$ & $55 \cdot 7$ & $63 \cdot 7$ & 55.9 \\
\hline $\mathrm{M}: \mathrm{F}$ & $22: 29$ & $9: 15$ & 13:14 & $4: 6$ \\
\hline Aspartate aminotransferase ${ }^{\star}$ & $4 \cdot 4$ & $4 \cdot 2$ & $4 \cdot 6$ & $3 \cdot 7$ \\
\hline Alkaline phosphatase & $5 \cdot 2$ & $4 \cdot 8$ & $5 \cdot 7$ & $4 \cdot 1$ \\
\hline$\gamma$ Glutamyltransferase $e^{\star}$ & 8.5 & 8.9 & $8 \cdot 1$ & 10 \\
\hline Bilirubin $^{\star}$ & 10 & 11 & $8 \cdot 8$ & $9 \cdot 4$ \\
\hline
\end{tabular}

^Enzyme and bilirubin values are given as a factor times the upper limit of normal concentration. Upper normal limits are $35 \mathrm{U} / \mathrm{l}$ for aspartate aminotransferase, $120 \mathrm{U} / \mathrm{l}$ for alkaline phosphatase, $30 \mathrm{U} / \mathrm{l}$ for $\gamma$ glutamyltransferase, and $18 \mu \mathrm{mo} / /$ for bilirubin.

ADRAC = Australian Drug Reactions Advisory Committee.

TABLE II-Crude odds ratios for developing flucloxacillin associated jaundice

\begin{tabular}{|c|c|c|c|}
\hline Variable & $\begin{array}{c}\text { No of cases } \\
(n=51)\end{array}$ & $\begin{array}{l}\text { No of controls } \\
\quad(n=199)\end{array}$ & $\begin{array}{c}\text { Odds ratio }(95 \% \\
\text { confidence interval) }\end{array}$ \\
\hline Age (years): Mean $\ddagger$ & $59 \cdot 8$ & $38 \cdot 3$ & - \\
\hline$<30$ & 3 & 84 & 1.00 \\
\hline $30-55$ & 17 & 58 & $7 \cdot 56(2.14$ to $26 \cdot 67)$ \\
\hline$>55$ & 31 & 54 & $18 \cdot 61(5 \cdot 16$ to $67 \cdot 17)$ \\
\hline \multicolumn{4}{|c|}{ Duration of treatment (days): $\neq$} \\
\hline$\leqslant 14$ & 32 & 180 & 1.00 \\
\hline$>14$ & 18 & 19 & $7 \cdot 13(2.90$ to 17.58$)$ \\
\hline Mean $(\mathrm{SD})$ & $14 \cdot 0(11 \cdot 3)$ & $8 \cdot 1(5 \cdot 5)$ & \\
\hline \multicolumn{4}{|c|}{ Previous serious illness or disease: } \\
\hline No & 20 & 131 & 1.00 \\
\hline Yes & 31 & 68 & $2.90(1.5$ to 5.9$)$ \\
\hline \multicolumn{4}{|l|}{ Concominant drugs: } \\
\hline No & 11 & 87 & 1.00 \\
\hline Yes & 40 & 112 & $2.80(1.3$ to 6.2$)$ \\
\hline \multicolumn{4}{|l|}{ Sex: } \\
\hline $\mathbf{M}$ & 29 & 86 & 1.00 \\
\hline $\mathrm{F}$ & 22 & 113 & $0.55(0.28$ to 1.06$)$ \\
\hline Mean (SD) dose (g): & $2.57(1.63)$ & $2 \cdot 18(1.53)$ & $p=0.16 t$ \\
\hline \multicolumn{4}{|c|}{ Indication for flucloxacillin: $\ddagger$} \\
\hline Prophylaxis & 14 & 50 & 1.00 \\
\hline Infection & 37 & 145 & $0.93(0.43$ to 1.94$)$ \\
\hline \multicolumn{4}{|l|}{ Smoker: $¥$} \\
\hline No & 17 & 67 & 1.00 \\
\hline Yes & 6 & 32 & 0.76 (0.28 to 2.07$)$ \\
\hline \multicolumn{4}{|l|}{ Drinks alcoholf } \\
\hline No & 6 & 36 & 1.00 \\
\hline Yes & 13 & 34 & $2.15(0.64$ to 7.26$)$ \\
\hline \multicolumn{4}{|l|}{ Excess alcohol intakeł } \\
\hline No & 19 & 67 & 1.00 \\
\hline \multirow{2}{*}{\multicolumn{4}{|c|}{$\begin{array}{l}\text { Yes } \\
\text { Allergy to penicillin } \neq\end{array}$}} \\
\hline & & & \\
\hline No & 43 & 168 & 1.00 \\
\hline Yes & 1 & 4 & $0.00(0.00$ to 4.00$)$ \\
\hline \multicolumn{4}{|l|}{ Previous flucloxacillin } \\
\hline No & 20 & 76 & 1.00 \\
\hline Yes & 3 & 21 & $0.64(0.16$ to 2.49$)$ \\
\hline \multicolumn{4}{|l|}{ General anaesthetic $\ddagger$} \\
\hline No & 45 & 177 & 1.00 \\
\hline Yes & 5 & 21 & $0.93(0.28$ to 3.07$)$ \\
\hline \multicolumn{4}{|l|}{$\begin{array}{l}\text { Yes } \\
\text { Route of administration }\end{array}$} \\
\hline Oral & 40 & 154 & 1.00 \\
\hline Intravenous & 6 & 16 & $1.40(0.40$ to 4.94$)$ \\
\hline Both & 5 & 29 & $0.69(0.19$ to 2.43$)$ \\
\hline
\end{tabular}

^Estimates of odds ratios for cases and matched controls obtained by conditional logistic regression.

†Two tailed Student's $t$ test.

$\ddagger$ Data not available on all patients.
TABLE III-Medical history of cases and controls taking flucloxacillin

\begin{tabular}{lcc}
\hline Type of medical condition & $\begin{array}{c}\text { No (\%) of cases } \\
(\mathrm{n}=51)\end{array}$ & $\begin{array}{c}\text { No }(\%) \text { of controls } \\
(\mathrm{n}=199)\end{array}$ \\
\hline Cardiac & $23(45)$ & $53(27)$ \\
Respiratory & $8(16)$ & $12(6)$ \\
Arthritis & $8(16)$ & $22(11)$ \\
Hepatitis & $3(6)$ & $3(2)$ \\
Gastrointestinal & $4(8)$ & $12(6)$ \\
Renal & $4(8)$ & $3(2)$ \\
Cancer & $3(6)$ & $6(3)$ \\
Dermatological & $4(8)$ & $1(1)$ \\
Neurological & $3(6)$ & $10(5)$ \\
Miscellaneous & $6(12)$ & $10(5)$ \\
\hline
\end{tabular}

TABLE IV-Drugs taken concurrently with flucloxacillin

\begin{tabular}{lcc}
\hline Drugs & $\begin{array}{c}\text { No (\%) of cases } \\
(\mathrm{n}=51)\end{array}$ & $\begin{array}{c}\text { No (\%) of controls } \\
(\mathrm{n}=199)\end{array}$ \\
\hline Phenoxymethylpenicillin & $10(20)$ & $16(8)$ \\
Amiloride plus hydrochlorothiazide & $5(10)$ & $4(2)$ \\
Heparin sodium & $7(14)$ & $9(5)$ \\
Digoxin & $5(10)$ & $5(3)$ \\
Temazepam & $7(14)$ & $17(9)$ \\
Salbutamol & $6(12)$ & $5(3)$ \\
Potassium supplements & $9(18)$ & $10(5)$ \\
Paracetamol & $10(20)$ & $29(15)$ \\
\hline
\end{tabular}

Table includes only drugs taken by at least five patients.

the relevant interaction (that is, cross product) terms in the conditional logistic regression models.

The protocol was approved by the ethics committee at each of the Victorian hospitals that participated in the study. The study was conducted according to the guidelines for the protection of privacy approved by the Australian privacy commissioner.

\section{Results}

Fifty one cases of flucloxacillin associated jaundice were studied, 24 of which were identified through the Adverse Drug Reaction Advisory Committee and 27 through the surveillance programme in Melbourne. Sixteen of the patients had been prescribed flucloxacillin in hospital and the remainder had been prescribed the drug by a private doctor.

Table I gives the characteristics of the patients identified from the two sources and those of patients in whom flucloxacillin was the sole suspected drug. No differences were observed by analysis of variance and the cases were amalgamated to form a single series.

Table II shows the characteristics of the cases and controls and the crude measures of association. In general cases were older and had received flucloxacillin for longer. Cases were more likely to have taken other prescribed drugs and experienced multiple underlying illnesses. However, no specific drug or underlying condition occurred substantially more commonly in cases than in controls (tables III and IV).

Several of the factors associated with flucloxacillin jaundice were also likely to be associated with one another. Age was the strongest associated factor and duration of treatment was also associated with risk of jaundice. Medical history and taking of concomitant drugs were also significantly associated with the risk of jaundice, but these associations disappeared after controlling for age.

An examination of the joint effect of age and duration of treatment indicated a significant interaction $(p=0.046)$ between the two effects (table V). Specifically, the effect of a longer duration of treatment was greater in the younger group (age $\leqslant 55$ ) than the older group (age $>55$ ).

\section{Discussion}

In Australia flucloxacillin is widely regarded as the best treatment for infections with methicillin sensitive 
TABLE v-Combined effect of age and duration of treatment on risk of flucloxacillin associated jaundice

\begin{tabular}{|c|c|c|c|}
\hline & $\begin{array}{l}\text { No of } \\
\text { cases } \\
(n=50)\end{array}$ & $\begin{array}{c}\text { No of } \\
\text { controls } \\
(n=196)\end{array}$ & $\begin{array}{c}\text { Odds ratio } \\
\text { (95\% confidence } \\
\text { interval) }\end{array}$ \\
\hline \multicolumn{4}{|l|}{ Age $\leqslant 55$ years: } \\
\hline$\leqslant 14$ Days' treatment & 12 & 133 & $1 \cdot 0^{\star}$ \\
\hline$>14$ Days' treatment & 8 & 9 & $13.3(3.7$ to 47.8$)$ \\
\hline \multicolumn{4}{|l|}{ Age $>55$ years: } \\
\hline$\leqslant 14$ Days' treatment & 20 & 44 & $6.0(2.4$ to 15.1$)$ \\
\hline$>14$ Days' treatment & 10 & 10 & $17.0(5.0$ to 57.8$)$ \\
\hline
\end{tabular}

${ }^{\star}$ Reference group is age $\leqslant 55$ years of age and duration of treatment of $\leqslant 14$ days.

Staphylococcus aureus and Staphylococcus epidermidis. It has almost replaced cloxacillin since it was found to be better absorbed after oral administration and to produce higher and more prolonged blood antibiotic concentrations. ${ }^{6}$ Flucloxacillin's increasing use has been accompanied, since 1989 , by a sharp increase in reports of severe and prolonged cholestatic jaundice, which has led to concern about the risk-benefit ratio for this drug. The condition has also been reported increasingly commonly in Sweden and to a lesser degree in the United Kingdom. ${ }^{4}$

We found that increasing age and prolonged duration of treatment are powerful risk factors for developing flucloxacillin associated jaundice. Concomitant drugs and previous serious illness were also associated but these reflected the older age of the cases and the relations disappeared once age was taken into account. There was no apparent relation with sex, route of administration, daily dose, or a history of penicillin allergy. The small size of the study did not allow precise estimation of the relative risk of jaundice for particular subgroups.

The study may have been affected by several types of bias. The cases probably represented an atypical subset of all patients with this condition because jaundice is more likely to be attributed to a drug if it is relatively severe and occurs soon after the drug is administered. However, this phenomenon would only affect our results if the factors associated with jaundice in unrecognised cases were different from those in the cases we studied.

Similarly, the controls were unlikely to be entirely representative of all subjects who received flucloxacillin without developing jaundice. Patients seen by the same prescribing doctor are a convenient control group but they may be more similar in terms of age, drug doses used, conditions treated, etc than would otherwise be expected. This effect would probably reduce the size of the odds ratios and thereby underestimate the strength of the relations observed.

Our findings are partly in keeping with those of Olsson et al, who studied 32 cases of flucloxacillin associated jaundice reported to the Swedish Adverse Drug Reactions Advisory Committee. ${ }^{5}$ Characteristics of these patients were compared with those of patients included in prescription sample surveys, which are regularly undertaken in Sweden. Patients developing jaundice were found to be older, to have received higher doses of flucloxacillin, and to be more likely to be female than those from the survey.

\section{RISK-BENEFIT RATIO}

The identification of subjects at increased risk of developing a serious adverse drug reaction can improve the risk-benefit ratio of drug treatment. For example, the finding that old age was a powerful risk factor for neutropenia after treatment with mianserin led to a warning about using this drug in elderly patients. ${ }^{7}$ However, the drug is still used widely in young people, where its low risk following overdose is an advantage. A similar strategy has been used with cotrimoxazole.

The percentage of cases of flucloxacillin associated jaundice attributable to advanced age can be determined by the epidemiological formula for population attributable risk. In 1991 in Australia 16\% of community prescriptions for flucloxacillin were for patients aged over 55 years (International Medical Statistics data, personal communication). The odds ratio for patients aged over 55 years (compared with those younger than 55 ) was $4 \cdot 2$. From these data it can be determined that one third of the cases which occurred outside hospital could be avoided if the drug were to be used exclusively in subjects under 55 years.

Flucloxacillin is a member of the isoxazolyl group of penicillins, which also includes cloxacillin, oxacillin, and dicloxacillin. In the United States, where flucloxacillin is not approved for use, no problem of similar size has been identified with the other isoxazolyl penicillins. Throughout the United States dicloxacillin (and to a lesser extent cloxacillin) is used for oral antistaphylococcal therapy while oxacillin is commonly used for parenteral treatment (United States Food and Drug Administration, personal communication). Each of these drugs has been reported to cause some degree of hepatotoxicity but their relative incidence is not clear. Olsson et al related the number of spontaneous reports of hepatotoxicity received by Swedish authorities to a measure of use (defined daily doses) and found a ratio of 1.9 cases per million defined daily doses with flucloxacillin, 1.3 with cloxacillin, and 0.75 with dicloxacillin. ${ }^{5}$ However, they gave little information about the severity of the hepatitis caused by each drug. Although it seems unlikely that these drugs could be associated with a hepatitis as severe as that associated with flucloxacillin, the possibility remains that the reaction has not been recognised.

In summary, this study identified increasing age and duration of treatment as powerful risk factors for the development of flucloxacillin associated jaundice. Because of the severity of this condition the drug should be prescribed only where there is a clear indication for antibiotics. When an older patient requires treatment or when prolonged treatment is likely, an alternative drug such as cloxacillin, oxacillin, or dicloxacillin should be considered. Careful prospective studies will be necessary to establish the hepatic risk associated with these drugs.

We thank the Adverse Drug Reactions Advisory Committee (ADRAC), the Melbourne liver group (in particular, $\mathrm{Dr}$ Sievert, Dr Gibson), Professor Yeomans, Dr Ross Bradbury, and Dr Jakobovits for their help and cooperation. Funding for this project was supplied by Commonwealth Serum Laboratories Limited and SmithKline Beecham.

1 Turner IB, Eckstein RP, Riley JW, Lunzer MR. Prolonged hepatic cholestasis after flucloxacillin therapy. Med $\mathcal{F}$ Aust 1989;151:701-4

2 Miros M, Kerlin P, Walker N, Harris O. Flucloxacillin induced delayed cholestatic hepatitis. Aust NZ J Med 1990;20:251-3.

3 Lobatto S, Dijkmans BAC, Mattie H, Van Hooff JP. Flucloxacillin-associated liver damage. Neth $\mathcal{F}$ Med 1982;25:47-8.

4 Fairley CK, Boyd I, Purcell P, McNeil JJ. Flucloxacillin jaundice. Lancet 1992;339:679.

5 Olsson R, Wiholm BE, Sand C, Zettergren L, Hultcrantz R, Myrhed M. Liver damage from flucloxacillin, cloxacillin, and dicloxacillin. $f \mathrm{Hepatol}$ 1992;15:154-61.

6 Sutherland R, Croydon EA, Rolinson GN. Flucloxacillin, a new isoxazolyl penicillin, compared with oxacillin, cloxacillin and dicloxacillin. $B M \mathcal{F}$ 1970;iv:455.

7 Coulter DM, Edwards RI. Mianserin and agranulocytosis in New Zealand. Lancet 1990;336:785-7.

(Accepted 12 November 1992) 